\title{
Entwicklungspolitische Gedanken zum Problemkreis Umwelt und Entwicklung im Vorfeld der UNCED
}

\section{Anne Bichsel}

\section{(2) OpenEdition Journals}

Electronic version

URL: http://journals.openedition.org/sjep/1381

DOI: $10.4000 /$ sjep.1381

ISSN: 1663-9677

\section{Publisher}

Institut de hautes études internationales et du développement

\section{Printed version}

Date of publication: 1 janvier 1992

Number of pages: 189-197

ISSN: $1660-5926$

\section{Electronic reference}

Anne Bichsel, «Entwicklungspolitische Gedanken zum Problemkreis Umwelt und Entwicklung im Vorfeld der UNCED », Schweizerisches Jahrbuch für Entwicklungspolitik [Online], 11 | 1992, Online erschienen am: 19 Mai 2013, abgerufen am 08 September 2020. URL : http://journals.openedition.org/ sjep/1381 ; DOI : https://doi.org/10.4000/sjep.1381 


\title{
Entwicklungspolitische Gedanken zum Problemkreis Umwelt und Entwicklung im Vorfeld der UNCED
}

\author{
Anne Bichsel
}

\section{Die Grenzen der Natur sind überschritten}

Die Warnsignale, dass die Grenzen der Natur überschritten werden, sind deutlich: Klimawandel aufgrund übermässiger Emissionen treibhauswirksamer Gase, die Zerstörung der schützenden Ozonschicht, Waldsterben, die chemische Verseuchung der Gewässer und Böden, gefährlich hohe Ozonwerte in bodennahen Luftschichten, Unmengen von nicht entsorgbarem Giftmüll, Verlust der Artenvielfalt. Das Unbehagen unter der Bevölkerung des Nordens wăchst von Tag zu Tag. Wissenschaftliche Erkenntnisse belegen, dass die Wirtschaftsweise des Nordens - die bisher als Entwicklungsmodell für den Rest der Welt galt- die Umwelt über die Regenerationsfähigkeit der Natur hinaus belastet. Sie muss deshalb umorientiert werden.

Die Atmosphäre und die Ozeane kennen keine nationalen Grenzen. Die Menschen im Süden sind ebenso wie jene im Norden von den Umweltschäden globaler Natur betroffen. Nur haben wirtschaftlich schwache Bevölkerungsgruppen noch weniger Möglichkeiten, sich dagegen zu wehren. Das wachsende Wirtschaftsgefälle zwischen Nord und Süd und die fortdauernde Armut breiter Bevölkerungsschichten im Süden führen ebenfalls zu irreparablen Umweltschäden. Armut zwingt zu übermăssiger und unangepasster Bewirtschaftung marginaler Räume. Der Zwang zur Devisenbeschaffung führt manchen Staat des Südens wider besseren Wissens zur Plünderung von Ressourcen. Wird im Norden die künttige Lebensbasis kurzfristigen Gewinnen und übermässigem Konsum geopfert, wird sie im Süden durch den heutigen Überlebenskampf vernichtet. 
Vor diesem Hintergrund werden die nächsten Jahrzehnte in der nationalen und internationalen Politik unter einem neuen Zeichen stehen. Standen früher Spannungen zwischen Ost und West politisch im Vordergnund, werden jetzt die Umweltprobleme in den internationalen Verhandlungen an vorderster Front stehen. Internationale Beschlüsse werden nationale Taten verlangen. Auf den langen Weg zur Neuorientierung der Weltwirtschaft in Richtung dauerhafter Entwicklung wird die UN-Konferenz über Umwelt und Entwicklung (UNCED) im Juni 1992 ein nächster Meilenstein sein. Mit der Verabschiedung einer Klimakonvention, einer Konvention zur Erhaltung der biologischen Vielfalt und eines Abkommens zum Schutz der Wälder sowie mit dem Festhalten von Prinzipien zur nachhaltigen Entwicklung der Weltwirtschaft (Erd-Charta) und mit der Erarbeitung eines Aktionsplanes zu deren Implementierung ("Agenda 21") sollen die Weichen für die nächsten Jahrzehnte gestellt werden.

\section{Klimawandel}

\section{Die Auswirkungen des Klimawandels auf die Entwicklungsländer}

Im Vordergrund der Umweltprobleme von globaler Tragweite steht der von Menschen verursachte Klimawandel. Obwohl nur eine von vielen Bedrohungen, lässt sich anhand dieses Problemkreises die Verantwortung des Nordens zur Lösung des Problems und die Schicksalsgemeinschaft von Norden und Süden illustrieren.

Auch wenn Differenzen über das Ausmass der zu enwartenden klimatischen Veränderungen bestehen, ist sich die Wissenschaft einig: die globale Erwärmung kann nicht mehr verhindert, sondern bestenfalls vermindert werden. Grösste Sorgen bereiten die Veränderungen des Wasserhaushaltes. Eine jahreszeitliche und regionale Umverteilung der Niederschläge, eine Zunahme von Extremereignissen (Dürren, Zyklonen und Überschwemmungen) und ein Austrocknen der Böden aufgrund zunehmender Verdunstung sind zu erwarten. Das Ansteigen des Meeresspiegels bedroht die Mündungsgebiete grosser Flüsse und andere dichtbesiedelte Küstenstriche - über die Hälfte der Weltbevölkerung lebt in Küstennähe. Weiter wird durch die Erhöhung des Meeresspiegels Meerwasser ins Grundwasser küstennaher Gebiete eindringen - eine Bedrohung für die Trinkwasserversorgung und für die Landwirtschaft. Veränderungen der Meeresströmungen werden die Fischerei regional hart treffen. Wie schnell sich Wälder den veränderten klimatischen Bedingungen anpassen können bleibt fraglich. Die weltweite Getreideproduktion könnte einen Rückgang von 5 bis 20 Prozent erleiden.

Obwohl der Norden Hauptverursacher dieses Umweltproblems ist, werden die Länder des Südens davon viel stärker betroffen sein. Von den wirtschaftlichen Sektoren wird der landwirtschaftliche am stärksten vom Klimawandel tangiert. Die Wirtschaft in der Dritten Welt ist bedeutend abhängiger von der Landwirtschaft als die der Industrieländer. In den Entwicklungsländern trägt die Landwirtschaft im Schnitt mit 20 Prozent zum Volkseinkommen bei und in manchen Ländern des Südens leben über zwei Drittel der Bevölkenung von der Landwirtschaft (Hurta- 
do,1991, S.2). Auch sind Agrarerzeugnisse oft die Hauptdevisenbringer. Im Gegensatz zu einjährigen Pflanzen wie Weizen oder Reis bedeuten Kulturpflanzen mit einer langen Vegetationszeit, wie z.B. Kaffee, bedeutende, langfristige Investitionen. Solche Kulturpflanzen reagieren aber bereits empfindlich auf geringste klimatische Schwankungen. Auf einen Schlag könnten wichtige volkswirtschaftliche Investitionen in ganzen Regionen zerstört werden.

Infolge des Bevölkerungsdruckes und der Armut sind die Menschen in weiten Teilen der Entwicklungsländer zur Bewirtschaftung marginaler Räume gezwungen. Aber je marginaler die Anbaubedingungen sind, sei es in bezug auf Klima, Bodenbeschaffenheit oder Topographie, desto empfindlicher ist das Ökosystem. Klimatische Veränderungen bedeuten eine zusätzliche Belastung und beschleunigen die Zerstörung ohnehin degradierter Landschaften.

Teilweise kann den negativen Folgen globaler Erwärmung mit Anpassungsstrategien begegnet werden. Die Staaten des Nordens können es sich leisten, Bewăssenungssysteme auszuweiten, Deiche gegen Überflutung zu bauen oder nach wetterbedingten Naturkatastrophen wieder aufzubauen. Die Bauern des Nordens sind gegen Ernteausfall infolge von Dürre oder Überschwemmungen versichert oder können mit staatlicher Hiffe rechnen. Die leeren Staatskassen der Entwicklungsländer werden es diesen nicht erlauben, solche Strategien zu verfolgen.

\section{Strukturanpassungen für den Norden: Das eigene Haus in Ordnung halten}

Die Verantwortung für globale Erwärmung liegt eindeutig bei den Industrieländern. Der Viertel der Menschheit, der in den Industrieländern lebt, verursacht drei Viertel der treibhauswirksamen Emissionen. Die Schuld der Industriestaaten reicht weit in die Vergangenheit zurück. Seit Beginn des industriellen Zeitalters haben sie den Umweltkosten ihres Wirtschaftens nicht Rechnung getragen. Sie haften nicht nur für die gegenwärtig übermässigen Emissionen, sondern auch für deren kumulative Anreichenung über Jahrzehnte.

Der Ansatzpunkt für Veränderungen liegt deshalb vor allem im Norden. Über ökologische Strukturanpassungen müssen die Industrieländer ihren Ressourcenverbrauch und Schadstoffausstoss auf ein Mass reduzieren, welches allen Menschen in Nord und Süd zugestanden werden kann, ohne die Natur irreversibel zu verändern. Die Folgen des Klimawandels zwingen uns, unseren eigenen Lebensstil in Frage zu stellen.

Das erste Gebot ist: Das eigene Haus in Ordnung halten. Als Schritt in die richtige Richtung sind im Norden politische Bestrebungen im Gange, Umweltkosten zu internalisieren. Das Verursacherprinzip soll zum Tragen kommen. Die Einführung von Lenkungsabgaben, Ökosteuern und Emissionszertifikaten führt in Richtung einer nachhaltigen Wirtschaftsweise.

Da bei unserer Produktionsweise Energie eine Schlüsselrolle einnimmt, ist die Einführung einer Energie- bzw. CO2-Abgabe von zentraler Bedeutung zur Verănderung unserer Wirtschaftsweise. Mit der Einführung einer Energieabgabe ist der erste Schritt zur Internalisierung der Umweltkosten verwirklicht. Eine Ausdehnung 
auf andere Bereiche muss folgen: Abgaben auf Pestizide und Dünger, Massnahmen gegen die Auslagerung von verschmutzenden Produktionsstătten in den Süden, Unterbindung des Gittmülttourismus, Einbeziehen der Sozial- und Umweltkosten in die Preise für Rohstoffe aus dem Süden, usw.

Über eine ganzheitliche Neuorientierung der steuerlichen Belastung kann unsere Wirtschaft in umweltvertrăglichere Bahnen gelenkt werden. Enwünschte Tätigkeiten wie Arbeit und Sparen wären weniger und umweltschädliche Produkte und Verhaltensweisen mehr zu belasten. Die bevorstehende Revision der Finanzordnung für 1994 bietet die Gelegenheit, Absichtserklärungen zur Internalisierung der Umweltkosten in konkrete Taten umzusetzen. Aus dieser Perspektive ist die Einführung einer Energieabgabe sowie die Revision der Finanzordnung in der Schweiz entwicklungspolitisch sehr bedeutsam.

\section{Schutz der Wălder und der biologischen Vielfalt}

Wälder sind zur Absorption von $\mathrm{CO} 2$ und als Standorte biologischen Reichtums von globaler Bedeutung. Die Industrieländer fordern die Entwicklungsländer zum Schutz dieser Wälder auf. Bei den Verhandlungen stellen die Industrienationen die Funktion der Wälder als "Lunge" der Erde in den Vordergrund und betonen, dass die Brandrodung der Tropenwälder mit 20 Prozent zu den globalen CO2Emissionen beiträgt. Der Norden betrachtet die Errichtung von Naturreservaten sowie die Durchführung von Wiederaufforstungsprojekten im Süden als Hauptlösungsansätze zur Bewahrung der Wälder und der biologischen Vielfalt. Er verkennt dabei die Bedeutung der Tropenwälder als Lebens- und Wirtschaftsraum für Millionen von UreinwohnerInnen und anderen BewohnerInnen. Nicht nur sind diese Menschen auf den Wald für ihr Überleben angewiesen, sondern einzig sie verfügen über das zur nachhaltigen Bewirtschaftung solcher empfindlicher Ökosysteme erforderliche Wissen.

Die Drittweltstaaten weisen denn auch darauf hin, dass der Norden 75 Prozent der $\mathrm{CO} 2-\mathrm{Emissionen}$ verursacht und dass er seine eigenen Ressourcen, darunter auch den Wald, im Zuge seiner Industrialisienung dezimiert hat. Im letzten Jahrhundert haben die Industriestaaten ihrerseits Unmengen von $\mathrm{CO} 2$ bei der Holzkohleverbrennung zur Energiegewinnung freigesetzt und ihre Wälder dabei auf einen Bruchteil des ursprünglichen Bestandes reduziert. Die Industriestaaten verurteilen die Entwicklungsländer für Praktiken, die sie selber verübt haben.

Ein bedeutender Rückgang der Artenvielfalt hat auch im Norden stattgefunden: Intensive Landwirtschaft unter grossem Einsatz von Pestiziden und Dünger ist eine der Hauptursachen. Durch die Ausbreitung dieses westlichen Landwirtschaftsmodells werden auch im Süden die genetisch wertvollen, über Jahrhunderte von lokalen Gemeinschaften kultivierten Landsorten durch einheitliche $\mathrm{Hy}$ briden ersetzt. Gerne bezeichnen die Industriestaaten die genetische Vielfalt als Erbe der Menschheit und fordern den freien Zugang zu den reichen genetischen Ressourcen des Südens. Diese Forderung steht aber in krassem Widerspruch zu den Bestrebungen der Industrie des Nordens, lebende Materie zu patentieren und 
so Gene in Privatbesitz zu überühren. Die Schweiz sollte aus entwicklungspolitischer Sicht freien Zugang zu den Forschungsresultaten sichern und den Patentierungsbestrebungen opponieren.

Zur Lösung der Waldfrage hat Malaysia vorgeschlagen, dass eine zur Gewährleistung des ökologischen Gleichgewichtes minimal notwendige Waldflächenbedeckung für die Erde festgelegt werden soll. Jedes Land hätte eine bestimmte Waldflăche zu stellen. Wird von den Entwicklungsländern verlangt, mehr als ihren Anteil für das Allgemeinwohl der Weltgemeinschaft zu stellen, müssten sie für den Nutzungsverzicht bzw. die Nutzungseinschränkung angemessen entschädigt werden (Raghavan, 1991, S.4). Diese Position verdient auch seitens der Schweiz Unterstützung.

Zumindest die Schweizer Bergbevölkenung müsste Verständnis für das Anliegen der Tropenwaldländer und der BewohnerInnen dieser Waldgebiete haben. Im Rahmen der Vernehmlassung zu einer europäischen Alpenkonvention zum Schutz der Berggebiete bekunden sie:

"Das Berggebiet ist nicht nur ein Erholungsraum, sondern in erster Linie auch Wirtschaftsraum für die ansässige Bevölkerung. Deshalb darf die Konvention nicht einseitig dem Schutze, sondern muss auch der Förderung des Alpenraums dienen. ... Unilaterale Einschränkungen können nur akzeptiert werden, wenn eine vollumfängliche [d.h. finanzielle, A.B.] Abgeltung sichergestellt ist." (Theler, 1991, S. 27).

\section{Armut als Ursache von Umweltschăden}

So wie es eine vom Reichtum bedingte Umweltzerstörung gibt, gibt es eine armutsbedingte. Wirtschaftliche Not und Bevölkenungsdruck zwingen die Menschen des Südens zur Besiedlung marginaler Räume. Übermässige und oft unangepasste Bewirtschaftung zerstört das ohnehin labile Ökosystem. Die Erträge nehmen ab. Der Druck auf die Umwelt nimmt zu. Dies ist ein bekannter Teufelskreis. Zerstörte Landschaften erhöhen die Landflucht. Es entstehen riesige Metropolen mit Millionen bitter armer Menschen. Die leeren Staatskassen der Regierungen des Südens erlauben es nicht, die notwendigen Infrastrukturen zu erstellen. Zunehmender Verkehr, das Fehlen sanitärer Einrichtungen sowie industrielle Emissionen belasten die Umwelt übermässig.

Unmittelbare Probleme im eigenen Land sind für die Entwicklungslănder vordringlicher als die vom Norden betonten Umweltprobleme von globaler Tragweite. In inrem Bericht The Challenge to the South schildert The South Commission die Meinung des Südens deutlich:

"The damage so far done to the environment has been overwhelmingly caused by the pattern of economic development in the North. Poverty is, in contrast, at the root of environmental degradation in poor countries... Multilateral arrangements for protecting the environment should therefore recognize that poverty and underdevelopment must be overcome for the environment in the 
South to be protected and should have the removal of poverty in the South as a central objective. It would be wholly unacceptable if the North were to seek to force the South to choose between development and environmental protection .." (S. 48; Hervorhebungen von A.B.).

Armutsbedingter Umweltzerstörung kann erst Einhalt geboten werden, wenn die Grundursachen beseitigt sind. Zu den wichtigen Ursachen gehören die Strukturen des Welthandelssystems. Im letzten Jahrzehnt sind die Rohstoffpreise auf den internationalen Märkten kontinuierlich gesunken, während der Schuldenberg stetig gewachsen ist. Dazu sind die Preise für Importe aus dem Norden gestiegen. 1988 betrug der Nettoabfluss von kommerziellem Kapital aus allen verschuldeten Ländern der Dritten Welt in Richtung Norden rund 50 Milliarden Dollar (Durning 1989, S.35).

Einkommensverluste der Entwicklungsländer aufgrund des Protektionismus der Industrieländer belaufen sich auf das Doppelte der öffentlichen Entwicklungshilfe der Industriestaaten pro Jahr (GATT-Forum 1991, S.3). Die Länder des Nordens müssen ihre Märkte für die Produkte des Südens öffnen und bereit sein, Produktepreise zu bezahlen, die die Sozial- und Umweltkosten decken. Eine Umkehr des Nettokapitalflusses über Entschuldungsmassnahmen und gerechtere Handelsstrukturen sind unerlässlich, damit die Entwicklungsländer tragfăhige Volkswirtschaften entwickeln können.

UNCED kann nicht das Forum zur Lösung der Schuldenkrise oder zu einer grundlegenden Reform der internationalen Handelsstrukturen sein. Zumindest sollten die Konferenzteilnehmer aber explizite Empfehlungen zuhanden der zuständigen internationalen Organisationen (GATT, Weltbank, IWF usw.) erarbeiten. Denn es wäre widersinnig in anderen Gremien Entscheidungen zu treffen, die dem Ziel globaler nachhaltiger Entwicklung entgegenlaufen. Zum Beispiel dürfen im Rahmen der GATT-Verhandlungen Massnahmen zum Schutz der Umwelt in keiner Weise als nichttarifäre Handelshemmnisse betrachtet und das Inkraftsetzen von Umweltschutznormen so verhindert werden.

\section{Bereitstellung von zusătzlichen finanziellen Mitteln}

Werden Länder des Südens den negativen Folgen des Klimawandels ausgesetzt, so sind die Industrieländer nach dem Verursacherprinzip moralisch verpflichtet, die daraus entstehenden Kosten mitzutragen. Die finanziellen Mittel sind nicht als grosszügige Spenden, sondern als Kompensation für zugefügtes Leid zu betrachten. Dazu erfolgen sie aus Eigeninteresse, denn bieten weite Landstriche im Süden keine Existenzbasis mehr, werden Menschen auch in entfernten Gegenden, d.h. auch im Norden, mit Migrationswellen konfrontiert werden.

Damit der Süden die Fehler der Industrienationen nicht wiederholt und seinen wirtschaftlichen Nachholbedarf ökologisch sinnvoll gestalten kann, müssen inm die neusten energiesparsamen und umweltschonenden Technologien zugänglich 
sein. Noch ist aber keineswegs klar, welche Technologien naturverträglich sind. UNCED soll das Verabschieden von international anerkannten Richtlinien fordern. Die Entwicklungsländer müssen als gleichwertige Partner auftreten und selber bestimmen können, welche Technologien für sie richtig sind. Dazu muss ihnen der Aufbau von eigenen Forschungskapazitäten und Kontrollbehörden ermöglicht werden. Auch darf Technologietransfer keine Einbahnstrasse bleiben. Die Industrienationen können viel über nachhaltige Ressourcennutzung von den Urbevölkerungen und Bauerngemeinschaften des Südens lernen. Ihr Wissen muss nicht nur anerkannt, sondern sie müssen auch dafür angemessen entschädigt werden.

Substantielle zusătzliche Mittel zu den Entwicklungshilfeleistungen werden deshalb vom Norden zur Verfügung gesteltt werden müssen. In ihrem Bericht Our Own Agenda (1990: viii) schildern die lateinamerikanischen Länder die Sicht des Südens deutlich:

"...the industrial countries must demonstrate an equal committment to sharing the burden of cost in a manner commensurate with their contribution to environmental degradation and their substantial ability to support and implement new environmental and development policies. The level of burden should reflect the accumulated environmental debt for which the industrial countries are primarily responsible." (Hervorhebung von A.B.)

Die Schweiz sollte deshalb die angekündigte Steigerung der Entwicklungshilfe auf 0,40 Prozent des Bruttosozialproduktes raschmöglichst, spätestens aber bis zum Ende der nächsten Legislaturperiode (1995), venwirklichen.

Zur Finanzierung von Projekten zum Schutze des Klimas, der Artenvielfalt, der Ozonschicht und der Meere wurde 1990 eine "Globale Umweltfazilität" geschaffen. Dieser Fonds wurde der Weltbank gemeinsam mit UNEP und UNDP anvertraut. Verhandlungen im Rahmen der dritten Vorbereitungskonferenz zu UNCED deuten auf eine mögliche Erweiterung dieser Umweltfazilităt hin. Mehrfach sind sowohl die Entscheidungs- und Verwaltungsstrukturen als auch die Projekte der Weltbank von Stimmen aus dem Norden und aus dem Süden mit Recht kritisiert worden. In Weltbankprojekten geniesst Quantität oft Vorrang vor Qualität. Entscheidungen werden nicht demokratisch, sondern im Verhältnis zu den von einem Land bereitgestellten Mitteln getroffen.

Um ihre Glaubwürdigkeit bei den Entwicklungsländern und bei den betroffenen Bevölkerungsgruppen zurückzugewinnen, muss sich die Weltbank verändern. Gefordert werden mehr Transparenz, gründliche Umweltverträglichkeitsprüfungen, enge Zusammenarbeit mit den Betroffenen und den mit ihnen zusammenarbeitenden nichtstaatlichen Organisationen sowie Gleichberechtigung der Empfänger- wie der Geberländer bei Entscheidungen. Die Absichtserklärungen zu Verbesserungen im Umweltbereich seitens der Weltbank sind zu begrüssen. Da aber eine Selbstbeurteilung nie objektiv sein kann, muss UNCED bei der Weltbank Offenheit fordern, damit unabhängige Institutionen den Erfolg der Reformbestrebungen beurteilen können. 


\section{Eigenverantwortung der Entwicklungslănder}

Bisher war die Rede von der Verantwortung der Industrieländer. Die Entwicklungslănder tragen aber ebenso die Verantwortung, für Ordnung im eigenen Haus zu sorgen. Mancher Drittweltstaat investiert mehr in Militărausgaben und Prestigebauten als in das Bildungs- und Gesundheitswesen. In Amazonien sind es in erster Linie landlose Arbeiter auf der Suche nach einem Auskommen, die den Urwald durch Brandrodung zerstören. Landreform sowie eine wirksame Besteuerung der Reichen tun Not. Es ist ein offenes Geheimnis, dass in manchem Land Geld alle Türen offnet. Nur ein starker politischer Wille im Lande selbst kann Korruption wirksam beseitigen.

Die Länder des Südens sind nicht bereit, von aussen auferlegte Bedingungen, v.a. in den Bereichen Waldschutz und Erhaltung der biologischen Vielfalt, zu akzeptieren. Misstrauisch aufgrund des gegenwärtigen Machtgefälles zwischen Nord und Süd berufen sie sich auf ihr Recht, als Nationalstaaten souverän über ihre Ressourcen zu verfügen. Ein solcher Anspruch entbindet aber nicht von der Verantwortung, mit den anvertrauten Ressourcen behutsam umzugehen.

\section{Zu den Erwartungen an UNCED 92}

Umweltprobleme von globaler Bedeutung sind nicht nur eine Bedrohung, sondern auch eine Chance zur Verwirklichung einer solidarischen Welt, in der die Menschen die Grenzen der Natur respektieren und ihre Schätze gerecht miteinander teilen. Ob die Teilnehmer der UNCED diese Chance wahrnehmen werden und die Weichen für die nächsten Jahrzehnte in Richtung dauerhafter Entwicklung stellen werden, bleibt noch abzuwarten.

Der UNCED-Prozess hat aber bereits Positives bewirkt. In Anerkennung ihrer Bedeutung und ihres fundierten Wissens in den Bereichen Umwelt und Entwicklung hat die Generalversammlung der Vereinten Nationen das Einbeziehen von nichtstaatlichen Organisationen im UNCED-Prozess gefordert. Bisher konnten über 300 nichtstaatliche Organisationen aus Nord und Süd an den Vorbereitungskonferenzen teilnehmen. Während der engen Zusammenarbeit und dem Austausch von Erfahrungen haben in den verschiedensten Bereichen tätige Organisationen (Umwelt, Entwicklung, KonsumentInnen, Frauen, bedrohte Völker, religiöse Gruppen usw.) erkannt, dass ihre Grundanliegen in die gleiche Richtung zielen und dass sie zusammen stark sind und vieles in Bewegung bringen können. Auch in der Schweiz bilden sich Koalitionen, die das politische Gewicht dieser Organisationen stärken werden. Sowohl auf nationaler wie auf internationaler Ebene soll die Integration von volksnahen privaten Organisationen im politischen Entscheidungsprozess fortgesetzt werden. Wie es bereits zahlreiche Industrieund Entwicklungsländer getan haben, soll auch die Schweiz Fachleute aus Ummeltkreisen und aus den hiffswerken als Beobachter sowohl in ihre UNCED-Delegation als auch in die Delegationen zukünftiger Verhandlungen aufnehmen. 
Bereits steht fest, dass die "Agenda 21", das Aktionsprogramm zur Erreichung nachhaltiger Entwicklung, rechtlich nicht verbindlich sein wird. Wahrhaftig kein gutes Zeichen, dass grosse Taten folgen werden. Die Teilnehmer der UNCED sind aber Nationen. Nationen sind letztlich Individuen. So liegt es an uns allen, Druck auf unsere Politiker auszuüben, damit der Weg zu nachhaltiger Entwicklung im eigenen Land eingeschlagen wird.

\section{Zusammenfassung}

Die Wirtschaftsweise des Nordens, die bisher als Entwicklungsmodell für den Rest der Welt galt, belastet die Umwelt über die Regenerationsfähigkeit der Natur hinaus. Über ökologische Strukturanpassungen müssen die Industrieländer ihren Ressourcenverbrauch und Schadstoffausstoss auf ein Mass reduzieren, welches allen Menschen in Nord und Süd zugestanden werden kann, ohne die Natur irreversibel zu verändern.

Obwohl der Norden Hauptverursacher der Umweltprobleme von globaler Tragweite ist, werden die Länder des Südens davon viel stärker betroffen sein. Damit sie diesen Gefahren begegnen sowie ihren wirtschaftlichen Nachholbedarf ökologisch sinnvoll gestalten können, wird der Norden dem Süden substantielle, zusätzliche finanzielle Mittel zur Vertügung stellen müssen.

Damit die notwendige Neuorientierung der Weltwirtschaft in Richtung dauerhafter Entwicklung verwirklicht werden kann, muss an der UN-Konferenz über Umwelt und Entwicklung (UNCED) im Juni 1992 ein Konsens unter den Industrienationen sowie zwischen Nord und Süd über den zu beschreitenden Weg erreicht werden.

\section{Literaturverzeichnis}

Durning, A., "Poverty and the Environment: Reversing the Downward Spiral», Worldwatch Paper, n 92, Washington, D.C., Worldwatch Institute, November 1989.

GATT-Forum, Für eine soziale und umweltverträgliche Weltwirtschafts- und Handelsordnung, Bern und Lausanne, 1991

Hurtado, M.E., "Farmers Reap What Others Sow", Panoscope, $n^{8} 24$, London, Panos, May, 1991, pp. 2-3.

Latin American and Caribbean Commission on Development and Environment, Our Own Agenda, Washington and New York, Inter-American Development Bank and UNDP, 1990.

Raghavan, C., "Avoid Double Standards, UNCED Told", Third World Resurgence, $n^{2}$ 9., Penang, Third World Network, 1991, pp.3-4.

Theler, L., "Noch ganz nach Geld steht der Sinn der Bergler", Die Weltwoche, $n^{2}$ 31, Zürich, 1. August 1991, p. 27.

The South Commission, The Challenge to the South. An Overview and Summary of the South Commission Report, Genf, The South Commission, 1990. 